\title{
PLASMOX $^{\circledR}$, ein Plasma-Ultra- hochtemperaturverfahren zur umweltgerechten Entsorgung von Sonderabfällen, mit Stoff- und Energierückgewinnungs- potentialen, sowie endlagerfähiger Schlackenproduktion
}

\author{
Mathias R. Fünfschilling*
}

Die Entsorgung toxischer Abfälle, unter dem Sammelbegriff Sondermüll eingestuft, bleibt weiterhin kontrovers. Im OECDRaum, in den wichtigsten Industrieländern der Welt, liegt der Pro-Kopf-Ausstoss von Sondermüll bei $c a .100 \mathrm{~kg}$ p.a., in der Schweiz wegen geringer Schwerindustrie bei $c a .50 \mathrm{~kg}$, in der Summe bei $c a .300000$ $\mathrm{t}$ jährlich (Schätzungen 1987). Neueste Erhebungen mit erstaunlichen Zahlen aus der Region Basel deuten eher auf 400000 $500000 \mathrm{t}$ hin

Auch in der Schweiz gelangen noch immer Sonderabfälle, ob als Neu- oder aus Altlasten, an die Umwelt mangels geeigneter oder realisierbarer Entsorgungsanlagen. Die bislang praktizierte Ausfuhr zur Verklappung, Hochseeverbrennung, Zwischen- und Endlagerung vorab in Drittweltländern, wird aufgrund strenger Export- und Transitbewilligungen zunehmend schwieriger und zwingt ein Binnenland wie die Schweiz, besonders dezidiert zu handeln. Es sei in diesem Zusammenhang an die Bemühungen von Bundesrat Flavio Cotti erinnert, die UNO-GiftmüllKonvention speziell von Drittwelt-Ländern ratifiziert zu bekommen, um dem 'Sondermülltourismus' entgegenzuwirken. Die in der Folge aufgelaufenen, nicht mehr exportierbaren Abfallmengen führen bei Industrie, Gewerbe und öffentlichen $\mathrm{Be}$ trieben zu einem akuten Notstand.

Aufgrund der dringend notwendigen neuen und strengen Umweltgesetze hat man sich zur UVP bekannt. Dieses Verfahren kann sich aber möglicherweise zu einer Entsorgungsverhinderung entpuppen, da Einsprachen möglich sind und St. Floriansche Eigeninteressen kleiner Bevölkerungsschichten nach dem Motto: 'Um-

* Korrespondenz: M.R. Fünfschilling

MGC-Plasma AG

Hofackerstr. 24

CH-4132 Mutten weltschutz, umweltgerechte Entsorgung ja, aber nicht vor meiner Gartentür', Vorschub geleistet wird. Diese gesetzliche Regelung hat bisher Lösungsansätze verzögert.

Die schweizerische Industrie hat weltweit auf dem Gebiete Umwelttechnik Pionierleistungen erbracht. Zu erwähnen sei die Kehrichtverbrennungstechnik, die Rauchgaswäsche oder die Nassoxydation. Es muss also ein erklärtes Ziel sein, diese Tradition fortzusetzen und gestützt auf konsequente Forschung und Entwicklung höhere Technologien einzuführen.

Die Malaise im Umgang mit Sonderabfällen, katastrophale Zustände um Altlasten, haben Umweltschutzorganisationen wie Greenpeace, WWF u.a. auf den Plan gerufen, deren sach- und fachkundige Argumente angehört werden müssen (UVP).

\section{Dilution is not the Solution to Pollution}

Die zulässigen Grenzwerte der Schadstoffe im Abgas, z.B. nach LRV, TA Luft u.a., können heute teilweise deutlich unterboten werden. Katalytisch werden z.B. die Stickoxyde um $4 / 5$ reduziert. Die Konzentrationsreduktion muss aber unbedingt zu einer Verminderung des Schadstoffausstosses pro Masseneinheit des entsorgten $\mathrm{Gu}$ tes führen. Beim Vergleich verschiedener Entsorgungsverfahren ist dieser Wert massgebend. Auch die Gewässer dürfen nicht für eine verschwenderische Rauchgaswäsche, mit dem Rauchgasvolumen korrelierend, missbraucht werden.

Dass beim Einsatz von Primär- und Sekundärenergien besonders haushälterisch, effizient umgegangen werden muss, versteht sich von selbst. Eine Nachverglasung der Schlacke ist deshalb weder energietechnisch, wirtschaftlich noch ökologisch vertretbar. Wärmekraftkopplung mit hohem Wirkungsgrad, Prozessdampferzeugung oder andere Energiewiederverwertungen werden für thermische Entsorgungsanla-

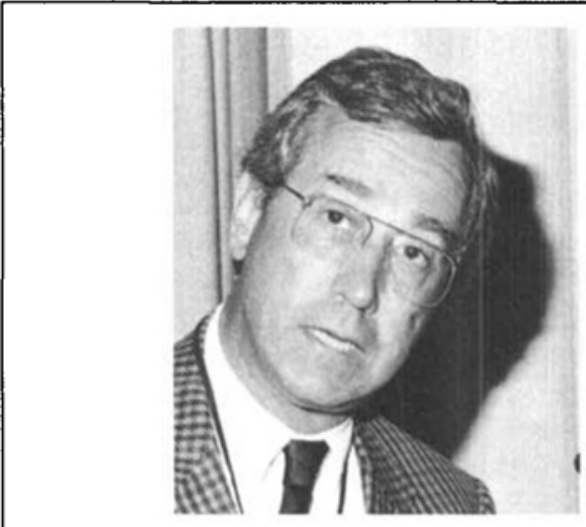

M.R. Fünfschilling: Geboren 1942, durchlief die Schulen in Basel und London. Nach dem MBAAbschluss am New York Institute of Finance folg. ten Volontariate in USA und Brasilien. Als Vorbereitung für die heutige Tätigkeit bei $M G C$ folgte eine Zusatzausbildung an der ETHZ, Abteilung Elektrotechnik. M.R. Fünfschilling hat sich Anfang der 80er Jahre insbesondere mit der Weiterentwicklung von $\mathrm{SF}_{6}$-isolierten Hochspannungswandlern beschäftigt, ab 1985 intensiv mit der Entwicklung der PLASMOX ${ }^{\star}$-Technologie, im joint venture mit Retech Inc., USA. M.R. Fünfschilling ist Vorsitzender der Geschäftsleitung Moser-Glaser \& Co. $A G$ sowie VR-Delegierter der $M G C$-Plasma $A G$. Seit 1987 ist er Präsident der Schweizerischen Elektrotechnischen Kommission.

gen als Selbstverständlichkeit vorausgesetzt. Die Energiebilanz muss jeder UVP standhalten.

Das Risk Management muss sich auch mit Fragen des Standortes, Anlagengrösse und -sicherheit, Zwischenlagerungen, Verarbeitungskapazität und Transportbewegungen auseinandersetzen. Wirtschaftlich wünschbare Grössen und Kapazitäten stehen zu oft diametral öffentlicher Akzeptanz gegenüber. Mit konventionellen Grossanlagen in der Schweiz sollten 4-5 überregionale Entsorgungszentren aufgebaut werden, welche wegen ihres Ausmasses aber in breiten Schichten der Öffentlichkeit nach wie vor auf Ablehnung stossen. Es muss deshalb das Ziel sein, mit höheren Technologien kleinere, kompakte Entsorgungseinheiten dort aufzubauen, wo der Sondermüll entsteht, und nicht das Gefahrenpotential, den Sondermülltransport mit zentralistischen Grossanlagen vergrössern. Auch können solche kleine Anlagen 'massgeschneidert', das heisst den jeweiligen Verhältnissen angepasst werden.

Es sei festgehalten, dass unsere heutigen Umweltprobleme, die absehbaren Umweltkrisen von morgen, nicht mit Technologien von gestern gelöst werden können. Das Plasma-Ultrahochtemperaturverfahren zeigt neue Wege zur umweltgerechten Entsorgung, auch zu einem neuen Schritt der Material- und Energierückgewinnung.

Bereits Ende des letzten Jahrhunderts war bekannt, dass Gase besondere Eigenschaften annehmen, wenn diese auf hohe Temperaturen gebracht werden. Bei $c a$. $2000^{\circ} \mathrm{C}$ lösen sich Gasmoleküle zu Atomen auf. Wird die Temperatur noch höher, 
über $3000^{\circ} \mathrm{C}$ gebracht, verlieren die Moleküle Elektronen, eine Ionisierung findet statt. Gase in diesem heissen, ionisierten Zustand werden elektrisch leitend, können in einem elektromagnetischen Feld kontrolliert, gesteuert werden und sind in einem Zustand von fast flüssiger Viskosität. Der deutsche Begriff Plasma deutet auf diesen Zustand hin. Temperaturen, gross genug um Gas in Plasma umzuformen, übersteigen normalerweise die Anforderungen für metallurgische Prozesse, wie zum Beispiel Eisen- und Stahlfertigung. Seit Beginn der Eisenzeit haben fossile Brennstoffe (Kohle) oder Biomasse (Holz) die Basisenergie zur Umwandlung von Erzen und Metallen geliefert.

Die aussergewöhnlichen Eigenschaften des Plasma, eingeschlossen das einmalige Energiepotential, hat Wissenschaftler und Industrieleute seit Jahrzehnten stark beschäftigt. Temperaturen, erzeugt mit fossilen Brennstoffen haben eine Limite von $2000^{\circ} \mathrm{C}$, elektrisch erzeugtes Plasma erreicht Temperaturen von $20000^{\circ} \mathrm{C}$ und mehr. Solche Temperaturgrössenordnungen öffnen die Türen zu unzähligen Möglichkeiten, viele sind noch nicht ausgeschöpft, speziell im Bereich der Materialverarbeitung. Schon anfangs des 20 . Jahrhunderts wurden Plasmareaktoren für die Herstellung von Chemikalien (Stickoxyde, Cyanwasserstoff, Acetylen etc.) verwendet. Plasma-Brenner wurden in den $50 \mathrm{er}$ Jahren verwendet, um Hitzeschilder für Wiedereintritt aus dem All zu prüfen. Das Verstehen komplexer Zusammenhänge und die Realisierung solcher Projekte haben wichtige Erkenntnisse gebracht. Sehr hohe Plasma-Temperaturen von $150 \mathrm{Mio}$. ${ }^{\circ} \mathrm{C}$ und mehr sind Voraussetzung für $\mathrm{Fu}$ sionsreaktoren, welche bekanntlich eines Tages unlimitierte Energie produzieren sollen.

In Anbetracht der Tatsache, dass Giftstoffabfälle ein grosses Entsorgungsproblem darstellen, speziell bei anfallenden Gemischen von Fest- und Flüssigstoffen, wurde 1987 mit der Entwicklung, Konstruktion und Bau einer Plasma-Anlage zur Zerstörung toxischer Abfälle begonnen. Die erste Industrieanlage ist im Rahmen des Superfund SITE Programms von der US-Umweltbehörde EPA in Butte/Mt in Betrieb genommen worden zur Entsorgung PCB-kontaminierter Erde sowie schwermetalldurchsetzter Schlämme. Nicht nur eine hohe DRE, sondern das Immobilisieren von Schwermetallrückständen in einer homogenen, nicht mehr auslaugbaren Schlacke soll grosstechnisch nachgewiesen werden.
Eine weitere $P L A S M O X^{\circledR}$-Anlage ist derzeit im Aufbau auf dem Werksgelände Moser-Glaser \& Co. $A G$ in Muttenz. Ziel der Phase I, ab 3. Quartal 1990, ist der experimentelle Industriebetrieb, wobei schwerpunktmässig drei wesentliche Ziele aus den 1987/88 erfolgreich erprobten Laborerkenntnissen heraus im Grossbetrieb bestätigt werden sollen:

1. Effiziente Entsorgung toxischer, schwermetallhaltiger Abfälle in flüssiger, pastöser und fester Form,

2. reststoffarme Rauchgase und Abwässer sowie deponiefähige Schlacke, das heisst keine Belastung der Umwelt,

3. Energierückgewinnung, Aufkonzentrierung und Rückgewinnung wertvoller Elemente.

\section{Das Verfahren}

Im Gegensatz zu den bekannten Verbrennungsmethoden werden beim PlasmaUltrahochtemperatur-Verfahren die Sonderabfälle in der ersten Stufe pyrolysiert, also nicht verbrannt. Speziell entwickelte Plasmabrenner erlauben enorm hohe Lichtbogentemperaturen und eine sehr hohe lokale Energiedichte. Die Temperaturskala reicht, je nach gewähltem Plasmagas, von $10000^{\circ} \mathrm{C}$ bis $20000^{\circ} \mathrm{C}$ oder mehr. Es ist also einerseits die hohe Lichtbogentemperatur und andererseits die hohe Energiedichte, die eine wirkungsvolle Zerstörung von Sonderabfällen ermöglichen. Es bildet sich dabei eine Schmelze aus, in der die schädlichen anorganischen Stoffe, wie zum Beispiel die Schwermetalle, eingebunden sind. Die Schmelze wird chargenweise abgelassen; sie verfestigt sich anschliessend zu einer Schlacke. Aus dem Pyrolyseprozess geht also in einem Verfahrensschritt eine konsolidierte, endlagerfähige Schlacke hervor. Sie zeigt beim EPAStandard-Leach-Test ausserordentlich gute Resultate. Die Schlacke lässt sich unter dem Begriff Inertstoff einreihen; ein Lagern auf Sonderdeponien ist somit nicht notwendig, sie kann vielmehr als Füllstoff in der Bauindustrie wiederverwertet werden.

Die aus dem Pyrolyseprozess hervorgehenden sehr geringen Abgasmengen werden in der zweiten Stufe, der Oxydationskammer, durch tangential angeordnete Sauerstoffjets oxydiert. Sie durchlaufen anschliessend eine dreistufige RauchgasNasswäsche mit Entstickung (DENOX $\left.{ }^{\circledR}\right)$. Bedingt durch das geringe Abgasvolumen kann die Rauchgasreinigungsanlage entsprechend klein dimensioniert werden. Die aus der Rauchgasreinigung und Abwasseraufbereitung anfallenden Schlämme werden in die Plasma-Anlage zurückgeführt und aufkonzentriert. Wertvolle, aufkonzentrierte Elemente können recycliert werden, nichtwiederverwertbare Substanzen werden in einer definierten Glasmatrix immobilisiert und als nichtauslaugbare Schlacke für Füllstoffe in die Baustoffindustrie oder auf Deponien überführt. Die Plasma-Anlage entsorgt sich selbst.

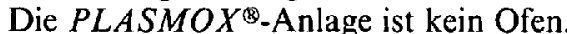
sondern ein geschlossenes Kaltwandsystem. Zum einen ist damit ein Austreten unerwünschter Nebenprodukte verunmöglicht, und zum anderen fallen auch keine kontaminierten Ofenauskleidungen an. Der einzige heisse Teil ist der Plasmalichtbogen. Damit entfällt auch ein Vorheizen der Anlage. Sie lässt sich beliebig einund ausschalten. Die Beschickung der Anlage erfolgt chargenweise mit 200-1-Normfässern. Die Gebinde werden gleich mitzerstört. Eine Nachreinigung entfällt. Kontaminiertes Erdreich, pumpbare Flüssigstoffe und Schlämme werden über einen Bypass direkt in den Plasmareaktor geführt.

Das PLASMOX $X^{\circledR}$-Verfahren eignet sich sowohl zur Zerstörung von flüssigen als auch von festen und pasteusen Sonderabfällen. Die Plasma-Anlagen lassen sich dank ihrer Modulbauweise entweder als On-line-Systeme in bestehende Produktionsprozesse eingliedern oder als Anlagen auf Zeit zur Entsorgung vor Ort konzipieren. Die Plasma-Anlagen können als selbständige Systeme oder auch ergänzend zu bestehenden thermischen Verfahren (Drehrohröfen) betrieben werden.

Wir sprechen von Entsorgung, von Aufbereitung unserer Neu- und Altlasten. Wir lassen uns dabei leiten von technischen, politischen, gesetzlichen und natürlich wirtschaftlichen Grundsätzen, auch Sachzwängen. Dabei vergessen wir immer wieder, dass wir die Verursacher unseres $\mathrm{Ge}$ genwartsproblemes Nr. 1 sind. Die Vergangenheit holt uns dann ein, wenn wir nicht sofort konsequente Abfallverminderung praktizieren und parallel dazu mit modernsten Entsorgungstechnologien die Energie- und Materialrekuperationen forcieren. Noch haben wir Rohstoffe. Die starke Wirtschaftsentwicklung sowie ein beängstigendes Bevölkerungswachstum zwingen uns, jetzt und richtig zu handeln. Ich fordere Sie auf, aus ihrer technischen und politischen Verantwortung heraus Ihre ganze Kraft in den Dienst der Abfallminderung, der Entsorgungstechnik und der Rekuperation zu stellen. 